\title{
ROTINA COMPUTACIONAL E EQUAÇÃO SIMPLIFICADA PARA MODELAR O TRANSPORTE DE SEDIMENTOS NUM LATOSSOLO VERMELHO DISTRÓFICO ${ }^{1}$
}

\section{GILMAR E. CERQUETANI², MARCÍLIO V. MARTINS FILHO ${ }^{3}$}

RESUMO: Os objetivos do presente trabalho foram desenvolver rotina computacional para a solução da equação de Yalin e do diagrama de Shields e avaliar uma equação simplificada para modelar a capacidade de transporte de sedimento num Latossolo Vermelho Distrófico que possa ser utilizada no Water Erosion Prediction Project - WEPP, assim como em outros modelos de predição da erosão do solo. A capacidade de transporte de sedimento para o fluxo superficial foi representada como função-potência da tensão cisalhante, a qual revelou ser aproximação da equação de Yalin. Essa equação simplificada pôde ser aplicada em resultados experimentais oriundos de topografia complexa. A equação simplificada demonstrou acuracidade em relação à equação de Yalin, quando calibrada utilizando-se da tensão média cisalhante. Testes de validação com dados independentes demonstraram que a equação simplificada foi eficiente para estimar a capacidade de transporte de sedimento.

PALAVRAS-CHAVE: erosão, modelagem, equação de Yalin.

\section{COMPUTATIONAL ROUTINE AND SIMPLIFIED EQUATION FOR MODELING SEDIMENT TRANSPORT CAPACITY IN A DYSTROPHIC HAPLUDOX}

\begin{abstract}
The objectives of the present work were to develop a computational routine to solve Yalin equation and Shield diagram and to evaluate a simplified equation for modeling sediment transport capacity in a Dystrophic Hapludox that could be used in the Water Erosion Prediction Project - WEPP, as well as other soil erosion models. Sediment transport capacity for shallow overland flow was represented as a power function of the hydraulic shear stress and which showed to be an approximation to the Yalin equation for sediment transport capacity. The simplified equation for sediment transport could be applied to experimental data from a complex topography. The simplified equation accurately approximated the Yalin equation when calibrated using the mean hydraulic shear stress. Validation tests using independent data showed that the simplified equation had a good performance in predicting sediment transport capacity.
\end{abstract}

KEYWORDS: erosion, modeling, Yalin equation.

\section{INTRODUÇÃO}

Diversos modelos de predição da erosão hídrica do solo utilizam a equação da continuidade aplicada ao transporte de sedimentos - um exemplo é o WEPP (Water Erosion Prediction Project). A erosão do solo no WEPP é descrita em termos dos processos de erosão em entressulcos e em sulcos, considerando-se a desagregação, a deposição, a tensão cisalhante, o escoamento nas áreas entressulcos e em sulcos, e a capacidade do fluxo de água em transportar sedimentos (NEARING et al., 1989):

$$
\begin{aligned}
& \frac{\partial q_{s}}{\partial x}=D_{f}+D_{i} \\
& D_{i}=C_{i} \quad K_{i} I^{2} S_{f} G_{e}\left(R_{s} / w\right)
\end{aligned}
$$

\footnotetext{
${ }^{1}$ Extraído da dissertação de Mestrado do primeiro autor.

${ }^{2}$ Eng ${ }^{\mathrm{o}}$ Agrônomo, Mestre em Agronomia, Coimbra-Frutesp Agroindustrial Ltda., Bebedouro - SP.

${ }^{3}$ Eng $^{\underline{0}}$ Agrônomo, Prof. Doutor, Departamento de Solos e Adubos, UNESP, Jaboticabal - SP, mfilho@ @cav.unesp.br.

Recebido pelo Conselho Editorial em: 22-11-2004

Aprovado pelo Conselho Editorial em: 14-7-2006
} 


$$
D_{f}=C_{r} K_{r}\left(\tau-\tau_{c r}\right)\left(1-G / T_{c}\right)
$$

em que,

$\partial \mathrm{q}_{\mathrm{s}} / \partial \mathrm{x}$ - taxa de transporte de sedimento por unidade de largura, $\mathrm{kg} \mathrm{s}^{-1} \mathrm{~m}^{-1}$;

$\partial \mathrm{x}$ - comprimento do declive, $\mathrm{m}$;

$\mathrm{D}_{\mathrm{f}}$ - taxa de erosão em sulcos, $\mathrm{kg} \mathrm{s}^{-1} \mathrm{~m}^{-2}$;

$\mathrm{D}_{\mathrm{i}}$ - taxa de erosão em entressulcos, $\mathrm{kg} \mathrm{s}^{-1} \mathrm{~m}^{-2}$;

$\mathrm{G}$ - carga de sedimento, $\mathrm{kg} \mathrm{m}^{-1} \mathrm{~s}^{-1}$;

$\mathrm{C}_{\mathrm{i}}$ - paramêtro de cobertura do dossel nos entressulcos, adimensional;

$\mathrm{K}_{\mathrm{i}}$ - paramêtro erodibilidade do solo entressulcos, $\mathrm{kg} \mathrm{m}^{-4} \mathrm{~s}^{-1}$;

$\mathrm{I}$ - intensidade da chuva, $\mathrm{m} \mathrm{s}^{-1}$;

$\mathrm{S}_{\mathrm{f}}$ - fator declividade em entressulcos, adimensional;

$\mathrm{G}_{\mathrm{e}}$ - cobertura efetiva da superfície do solo, adimensional;

$\mathrm{R}_{\mathrm{s}}$ - espaçamento entre os sulcos, $\mathrm{m}$;

$\mathrm{W}$ - largura dos sulcos, $\mathrm{m}$;

$\mathrm{C}_{\mathrm{r}}$ - parâmetro de cobertura dos sulcos, adimensional;

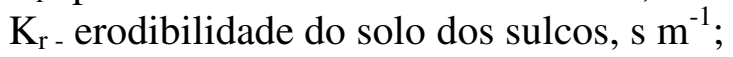

$\tau$ - tensão cisalhante, $\mathrm{Pa}$;

$\tau_{\mathrm{cr}}$ - tensão cisalhante crítica, $\mathrm{Pa}$, e

$\mathrm{T}_{\mathrm{c}}$ - capacidade de transporte de sedimento pelo fluxo, $\mathrm{kg} \mathrm{m}^{-1} \mathrm{~s}^{-1}$.

A eq.(2) decreve a erosão entressulcos, a qual decorre da desagregação das partículas do solo pelo impacto das gotas d'água e que são transportadas para os sulcos. Já a eq.(3) descreve a taxa de erosão do solo desagregado nos sulcos pela ação conjunta do escoamento e tensão cisalhante exercida pelo fluxo concentrado de enxurrada.

Segundo LU et al. (2001), caso $\mathrm{D}_{\mathrm{i}}$ seja considerada muito pequena, como nos casos em que a intensidade da chuva é baixa, a eq.(1) seria:

$$
\frac{\partial q_{s}}{\partial x}=D_{i}
$$

Considerando-se o não-efeito de cobertura em sulcos $\left(\mathrm{C}_{\mathrm{r}}=1\right)$, a eq. (3) pode ser reescrita como FOSTER et al. (1989):

$$
D_{f}=K_{r}\left(\tau-\tau_{c}\right)\left(1-\frac{G}{T_{C}}\right)
$$

O termo 1-G/T $\mathrm{T}_{\mathrm{c}}$, na eq.(5), representa um balanço da desagregação/deposição no decorrer de um evento, o qual demonstra que a taxa de erosão em sulcos é uma função da carga de sedimentos em relação à capacidade de transporte do fluxo. Portanto, conforme a eq.(5), a desagregação em sulcos ocorre apenas quando $\tau>\tau_{c}$ e se $G<T_{c}$, pois, nesse caso, $D_{f}$ é positiva $\left(G / T_{c}<1\right)$. Já a deposição de sedimentos ocorre quando $G>T_{c}$. Desse modo, $D_{f}$ é negativa $\left(G / T_{c}>1\right)$ e pode ser avaliada como:

$$
D_{f}=\left(\beta, V_{f} / q\right)\left(T_{c}-G\right)
$$

em que,

$\beta$ ' - coeficiente de turbulência induzida pelo impacto das gotas da chuva;

$\mathrm{V}_{\mathrm{f}}$ - velocidade efetiva das partículas do solo no fluxo de enxurrada, $\mathrm{m} \mathrm{s}^{-1}$, e

$\mathrm{q}$ - vazão do escoamento por unidade de largura do sulco, $\mathrm{m}^{-2} \mathrm{~s}^{-1}$.

Para uso do modelo WEPP, a determinação da capacidade de transporte de sedimentos $\left(\mathrm{T}_{\mathrm{c}}\right)$ é fundamental, visto ser ela, em associação com a carga de sedimentos $(G)$, fatores que coordenam a desagregação e a deposição de partículas. 
Muitos pesquisadores têm dado atenção para a determinação da capacidade de transporte de sedimentos (FOSTER, 1982; FINKNER et al., 1989; SHARMA et al., 1996; LEI et al., 2001; POLYAKOV \& NEARING, 2003). Várias fórmulas têm sido desenvolvidas para calcular a capacidade de transporte de sedimentos, mas nenhuma expressão e/ou método desenvolvidos tem ampla aceitação para fluxos superficiais e situações de sedimentação não-uniforme (LEI et al., 2001). Contudo, ALONSO et al. (1981) avaliaram que a equação de Yalin foi a mais apropriada para determinar $T_{c}$. A equação de Yalin é definida como (YALIN, 1963):

$$
\frac{\mathrm{T}_{\mathrm{C}}}{\mathrm{S}_{\mathrm{g}} \mathrm{d} \rho_{\mathrm{w}}{ }^{1 / 2} \tau^{1 / 2}}=0,635 \delta\left[1-\frac{1}{\beta} \ln (1+\beta)\right]
$$

$\operatorname{com} \beta$ e $\delta$ expressos como:

$$
\begin{aligned}
& \beta=2,45 \mathrm{~S}_{\mathrm{g}}^{-0,4} \mathrm{Y}_{\mathrm{cr}}^{0,5} \delta \\
& \delta=\frac{\mathrm{Y}}{\mathrm{Y}_{\mathrm{cr}}}-1 \quad \text { (quando } \mathrm{Y}<\mathrm{Y}_{\mathrm{cr}}, \delta=0 \text { ) }
\end{aligned}
$$

sendo,

$$
Y=\frac{V^{2}}{\left(S_{g}-1\right) g d}
$$

em que,

$\mathrm{S}_{\mathrm{g}}$ - densidade de partícula, assumida neste trabalho como igual a 2,65, adimensional;

d - diâmetro de partícula, m;

$\rho_{\mathrm{w}}$ - densidade da água, $\mathrm{kg} \mathrm{m}^{-3}$;

$\tau$ - tensão cisalhante atuando no leito ou fundo do canal, Pa;

Y - tensão cisalhante, adimensional;

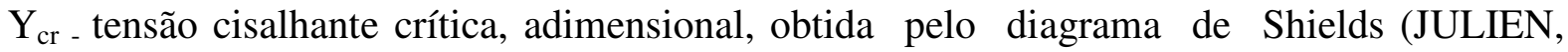
1998);

$\mathrm{V}$ - velocidade cisalhante, $\mathrm{m} \mathrm{s}^{-1}$

$\mathrm{g}$ - aceleração da gravidade, $\mathrm{m} \mathrm{s}^{-2}$, e

$\beta$ e $\delta$ - parâmetros adimensionais definidos pelas eqs.(8) e (9), respectivamente.

Uma simplificação da equação de Yalin foi apresentada por FINKNER et al. (1989), com o intuito de reduzir o número de cálculos necessários para obter $\mathrm{T}_{\mathrm{c}} \mathrm{e}$ aliviar as instabilidades associadas com as soluções numéricas. Segundo SHARMA et al. (1996), a equação de Yalin, em sua forma original [eqs.(7) a (10)], é um longo e complexo sistema de equações. Há, além do exposto, o fato de ser necessário o uso do diagrama de Shields (JULIEN, 1998) na resolução do referido sistema, o que também contribui com relativa morosidade para solucionar a equação de Yalin.

Desse modo, os objetivos deste estudo são desenvolver rotina computacional para solução da equação de Yalin e do diagrama de Shields e avaliar uma equação simplificada para modelar a capacidade de transporte de sedimento $\left(\mathrm{T}_{\mathrm{c}}\right)$ num Latossolo Vermelho Distrófico que possa ser utilizada no Water Erosion Prediction Project - WEPP, assim como em outros modelos de predição da erosão do solo.

\section{MATERIAL E MÉTODOS}

Uma rotina computacional para a determinação da capacidade de transporte de sedimentos $\left(\mathrm{T}_{\mathrm{c}}\right)$ foi gerada para solucionar a equação de Yalin, a qual foi designada MSEY (Modelo para Solução da Equação de Yalin). O MSEY exige como parâmetros de entrada a temperatura do fluxo (T), o diâmetro de partículas (d), a tensão cisalhante do fluxo $(\tau)$, a velocidade cisalhante do fluxo (V) e a densidade das partículas do sedimento $\left(S_{\mathrm{g}}\right)$. Internamente, a rotina MSEY calcula os 
parâmetros de saída necessários para a solução das eqs.(7) a (10) e do diagrama de Shields (JULIEN, 1998). Na Figura 1, apresenta-se o fluxograma final da rotina computacional MSEY, que foi desenvolvida numa planilha eletrônica, em ambiente Windows ${ }^{\mathrm{TM}}$, executada no Microsoft Excel 97® ou superior. O MSEY é composto por seis planilhas: O MSEY, ENTRADA DE DADOS, RESULTADOS, GRÁFICO, BASE 1 e BASE 2.

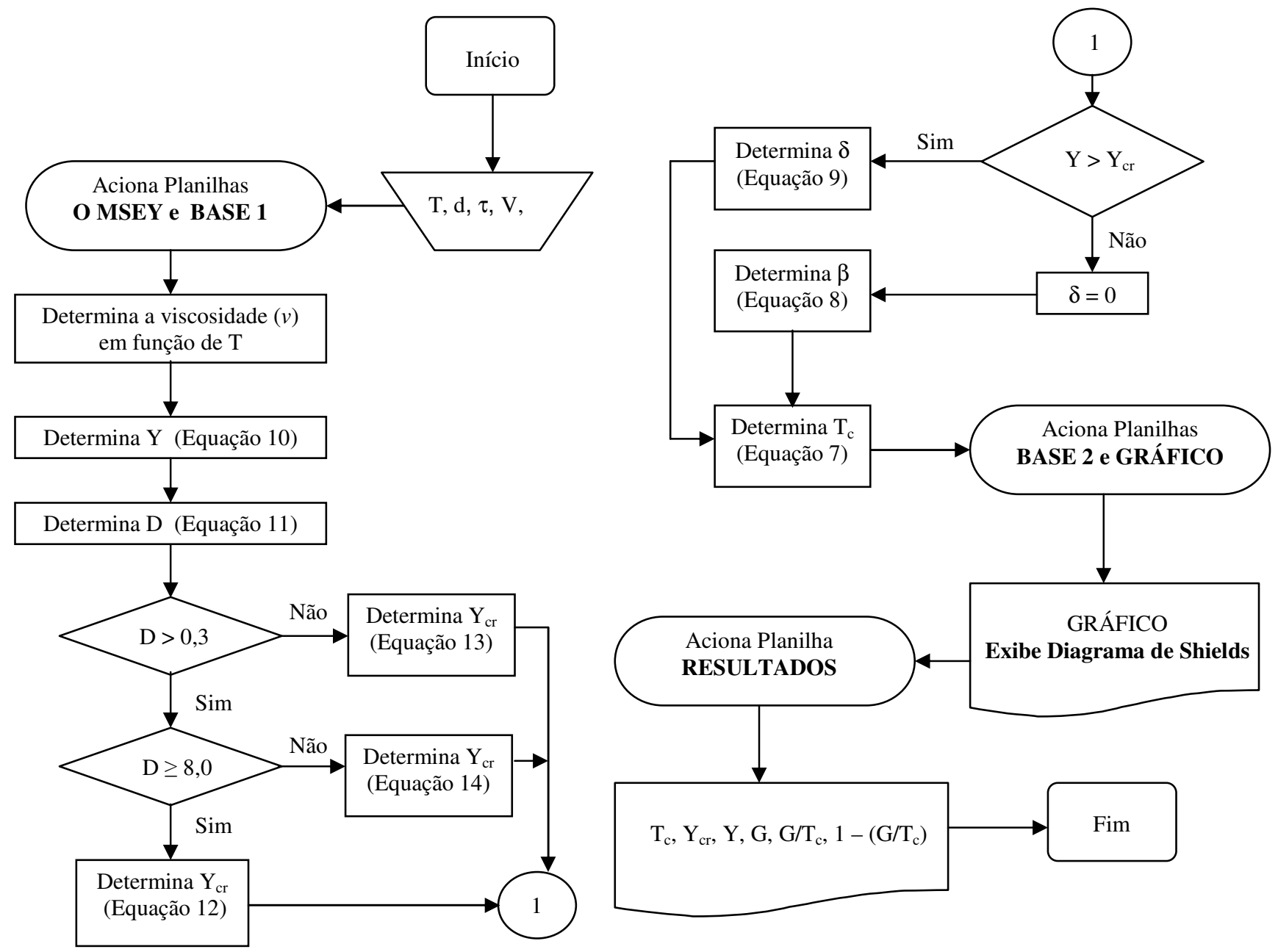

FIGURA 1. Fluxograma da rotina MSEY - Modelo para Solução da Equação de Yalin.

As planilhas O MSEY, RESULTADOS, GRÁFICO, BASE 1 e BASE 2 apresentam suas células protegidas. O usuário não pode alterar, modificar ou acrescentar informações aos seus conteúdos. A única planilha não-protegida é a ENTRADA DE DADOS.

O MSEY gera uma solução do diagrama de Shields e faz uma apresentação gráfica do mesmo, na qual posiciona a(s) partícula(s) em função do(s) seu(s) diâmetro(s) e Y. O referido posicionamento, no gráfico do MSEY, aponta se há ou não condições de a(s) partícula(s) com dado(s) diâmetro(s) iniciar (em) ou não o movimento. Desse modo, se $\mathrm{Y}>\mathrm{Y}_{\mathrm{cr}}$, o posicionamento indicará movimento e em caso contrário não. Para compor o diagrama de Shields, o MSEY utilizase das equações apresentadas por JULIEN (1998):

$$
\begin{aligned}
& \mathrm{D}=\left[\frac{\left(\mathrm{S}_{\mathrm{g}}-1\right) \mathrm{g}}{\mathrm{v}^{2}}\right]^{1 / 3} \mathrm{~d} \\
& \mathrm{Y}_{\mathrm{cr}}=\frac{0,23}{\mathrm{D}}+0,054\left[1-\exp \left(\frac{-(\mathrm{D})^{0,85}}{23}\right)\right] \quad(\text { para } \mathrm{D}>8,0)
\end{aligned}
$$




$$
\begin{array}{ll}
\mathrm{Y}_{\mathrm{cr}}=0,5 \tan \phi & (\text { para } \mathrm{D}<0,3) \\
\mathrm{Y}_{\mathrm{cr}}=0,25 \mathrm{D}-0,6 \tan \phi & (\text { para } \mathrm{D} \text { entre } 0,3 \text { e 8,0) }
\end{array}
$$

em que,

D - diâmetro de partícula, adimensional;

d - diâmetro de partícula, m;

$v$ - viscosidade cinemática da água, $\mathrm{m}^{2} \mathrm{~s}^{-1}, \mathrm{e}$

$\phi$ - ângulo de repouso e é igual a $30^{\circ}$ para partículas do sedimento nos padrões descritos para as eqs.(13) e (14).

Uma particular incoveniência do diagrama original de Shields é que a velocidade cisalhante $(V)$ e a tensão cisalhante $(\tau)$ são expressas no eixo das abscissas e no das ordenadas como variáveis dependente e independente, respectivamente. Como tais variáveis são intercambiáveis $\left(\mathrm{V}=\sqrt{\tau / \rho_{\mathrm{w}}}\right)$, isso pode causar dificuldades na interpretação do diagrama de Shields. Assim, o parâmetro $\mathrm{D}$ foi adotado para eliminar $\mathrm{V}$ no eixo das abscissas, como proposto por PAPHITIS (2001).

Resultados de temperatura do fluxo (T), diâmetro de partículas (d), tensão cisalhante do fluxo $(\tau)$, velocidade cisalhante do fluxo $(\mathrm{V})$ e densidade de partículas do sedimento $\left(\mathrm{S}_{\mathrm{g}}\right)$, obtidos em experimento sobre erosão em sulcos por MARTINS FILHO (1994), num Latossolo Vermelho distrófico, cujas faixas de variações são as apresentadas na Tabela 1, foram utilizados no MSEY para estabelecer valores da capacidade de transporte de sedimentos $\left(\mathrm{T}_{\mathrm{c}}\right)$ para 24 observações. Com esses valores, análises de regressão foram conduzidas com o programa STATISTICA (STATSOFT, 1994) com o intuito de calibrar equações entre valores de tensão cisalhante $(\tau)$ e $T_{c}$. Como critério para definir o processo de calibração, utilizou-se das equações com os maiores valores de coeficiente de determinação $\left(\mathrm{R}^{2}\right)$. Para a capacidade de transporte de sedimento $\left(\mathrm{T}_{\mathrm{c}}\right)$, o modelo simplificado de FINKNER et al. (1989) foi a base referencial para a calibração de equações, uma vez que esse foi proposto por FOSTER et al. (1989) para uso no WEPP. Tal modelo pode ser descrito como:

$$
\mathrm{T}_{\mathrm{c}}=\mathrm{K}_{\mathrm{t}} \tau^{\mathrm{z}}
$$

em que,

$\mathrm{K}_{\mathrm{t}}$ - coeficiente de transporte de sedimento;

$\tau$ - tensão cisalhante, $\mathrm{Pa}, \mathrm{e}$

z - expoente igual a 3/2 (FINKNER et al., 1989).

TABELA 1. Faixa de valores dos resultados obtidos por MARTINS FILHO (1994) e utilizados na rotina MSEY.

\begin{tabular}{ccccccc}
\hline $\mathrm{N}$ & $\mathrm{T}\left({ }^{\circ} \mathrm{C}\right)$ & $\mathrm{S}\left(\mathrm{m} \mathrm{m}^{-1}\right)$ & $\mathrm{w}(\mathrm{m})$ & $\tau(\mathrm{Pa})$ & $\mathrm{G} 10^{3}\left(\mathrm{~kg} \mathrm{~s}^{-1} \mathrm{~m}^{-1}\right)$ & $\mathrm{d}(\mathrm{m})$ \\
\hline 24 & 25 & 0,070 a 0,078 & 0,0174 a 0,2201 & 2,96 a 12,50 & 0,370 a 22,571 & 0,00019 a 0,00030 \\
\hline
\end{tabular}

N - número de observações; T - temperatura do fluxo; S - razão de inclinação; w - largura do canal; $\tau$ - tensão cisalhante; G - carga de sedimento; d - diâmetro de partícula.

$\mathrm{Na}$ avaliação das equações calibradas, utilizaram-se os resultados de erosão obtidos por PEREIRA (1989) em parcelas experimentais com 1,5 m de largura e comprimentos de declive variando de 1,0 a 8,0 m (Tabela 2). PEREIRA (1989) observou a presença de erosão em sulcos para comprimentos de rampa a partir de 1,0 m. Desse modo, foi necessária a conversão dos referidos resultados de macroparcelas para sulcos individuais. Isso foi conduzido de modo semelhante ao realizado por GILLEY \& DORAN (1998). Na referida conversão, foram utilizadas as seguintes equações (GILLEY et al., 1990 e GILLEY \& DORAN, 1998):

$$
\mathrm{R}_{\mathrm{n}}=\frac{\mathrm{q}}{v}
$$




$$
\begin{aligned}
& \mathrm{f}=1350 \mathrm{R}_{\mathrm{n}}{ }^{-0,934} \\
& \mathrm{y}=\left(\frac{\mathrm{f} \mathrm{q}^{2}}{8 \mathrm{~g} \mathrm{~S}}\right)^{1 / 3} \\
& \tau=y_{\mathrm{w}} \text { y } \mathrm{S}
\end{aligned}
$$

em que,

$\mathrm{R}_{\mathrm{n}}$ - número de Reynolds;

$\mathrm{q}$ - taxa de descarga por unidade de largura, $\mathrm{m}^{2} \mathrm{~s}^{-1}$;

$v$ - viscosidade cinemática da água, $\mathrm{m}^{2} \mathrm{~s}^{-1}$;

f - coeficiente de rugosidade de Darcy-Weisbach;

$\mathrm{y}$ - profundidade do fluxo, $\mathrm{m}$;

$\mathrm{g}$ - aceleração da gravidade, $\mathrm{m} \mathrm{s}^{-2}$;

$\tau$ - tensão cisalhante $(\mathrm{Pa})$;

$y_{\mathrm{w}}$ - peso específico da água $\left(\mathrm{N} \mathrm{m}^{-3}\right)$,

$\mathrm{S}$ - razão de inclinação, $\mathrm{m} \mathrm{m}^{-1}$.

TABELA 2. Faixa de valores dos resultados obtidos por PEREIRA (1989) e utilizados no teste de comparação de modelos.

\begin{tabular}{cccccc}
\hline $\mathrm{N}$ & $\lambda(\mathrm{m})$ & $\mathrm{S}\left(\mathrm{m} \mathrm{m}^{-1}\right)$ & $\mathrm{d}(\mathrm{m})$ & $\mathrm{G} 10^{3}\left(\mathrm{~kg} \mathrm{~s}^{-1} \mathrm{~m}^{-1}\right)$ & ${\mathrm{q}\left(\mathrm{m}^{2} \mathrm{~s}^{-1}\right)}$ \\
\hline 30 & 1,0 & 0,058 & 0,000191 a 0,000546 & 0,003 a 0,823 & 0,008 a 0,060 \\
30 & 2,0 & 0,066 & 0,000277 a 0,000625 & 0,052 a 0,216 & 0,008 a 0,056 \\
30 & 4,0 & 0,050 & 0,000534 a 0,000586 & 0,131 a 2,541 & 0,010 a 0,063 \\
30 & 8,0 & 0,106 & 0,000505 a 0,000629 & 0,176 a 7,601 & 0,007 a 0,246 \\
\hline
\end{tabular}

$\mathrm{N}$ - número de observações; $\lambda$ - comprimento do declive; $\mathrm{S}$ - razão de inclinação; $\mathrm{d}$ - diâmetro de partícula; $\mathrm{G}$ - carga de sedimento; q - taxa de descarga por unidade de largura.

Para testes de validação de modelos, utilizaram-se parâmetros estatísticos propostos por LOAGUE \& GREEN (1991), como:

$$
\begin{aligned}
& \mathrm{RMSE}=\sum_{\mathrm{i}=1}^{\mathrm{n}}\left[\left(\mathrm{P}_{\mathrm{i}}-\mathrm{O}_{\mathrm{i}}\right)^{2} / \mathrm{n}\right]^{0,5}\left(100 / \mathrm{O}_{\mathrm{m}}\right) \\
& \mathrm{EF}=\left[\sum_{\mathrm{i}=1}^{\mathrm{n}}\left(\mathrm{O}_{\mathrm{i}}-\mathrm{O}_{\mathrm{m}}\right)^{2}-\sum_{\mathrm{i}=1}^{\mathrm{n}}\left(\mathrm{P}_{\mathrm{i}}-\mathrm{O}_{\mathrm{i}}\right)^{2}\right] / \sum_{\mathrm{i}=1}^{\mathrm{n}}\left(\mathrm{O}_{\mathrm{i}}-\mathrm{O}_{\mathrm{m}}\right)^{2} \\
& \mathrm{CRM}=\sum_{\mathrm{i}=1}^{\mathrm{n}}\left(\mathrm{O}_{\mathrm{i}}-\mathrm{P}_{\mathrm{i}}\right) / \sum_{\mathrm{i}=1}^{\mathrm{n}} \mathrm{O}_{\mathrm{i}}
\end{aligned}
$$

em que,

RMSE - erro-padrão da estimativa normalizado, \%;

EF - eficiência do modelo;

CRM - coeficiente de massa residual;

$P_{\mathrm{i}}$ - valor estimado;

$\mathrm{O}_{\mathrm{i}}$ - valor observado;

$\mathrm{O}_{\mathrm{m}}$ - média dos valores observados, e

$\mathrm{n}$ - espaço amostral.

Considerou-se, como descrito por MARTINS FILHO (1999), que quando valores preditos e observados são iguais, os parâmetros estatísticos RMSE, EF e CRM assumem valores iguais a 0; 1 e 0 , respectivamente. 


\section{RESULTADOS E DISCUSSÃO}

Com base nos valores de MARTINS FILHO (1994), obtidos a partir de 24 observações, as quais foram resumidamente apresentadas na Tabela 1, determinou-se a capacidade de transporte de sedimentos $\left(\mathrm{T}_{\mathfrak{c}}\right)$ com a rotina MSEY. Na Figura 2, apresenta-se o resultado gráfico do diagrama de Shields na rotina MSEY, com o qual é verificada a transição da condição de não-movimento para início de movimento do sedimento. Essa condição é definida, segundo PAPHITIS (2001), como crítica ou limite de início de movimentação, a qual está relacionada com o primeiro estágio do transporte de sedimento.

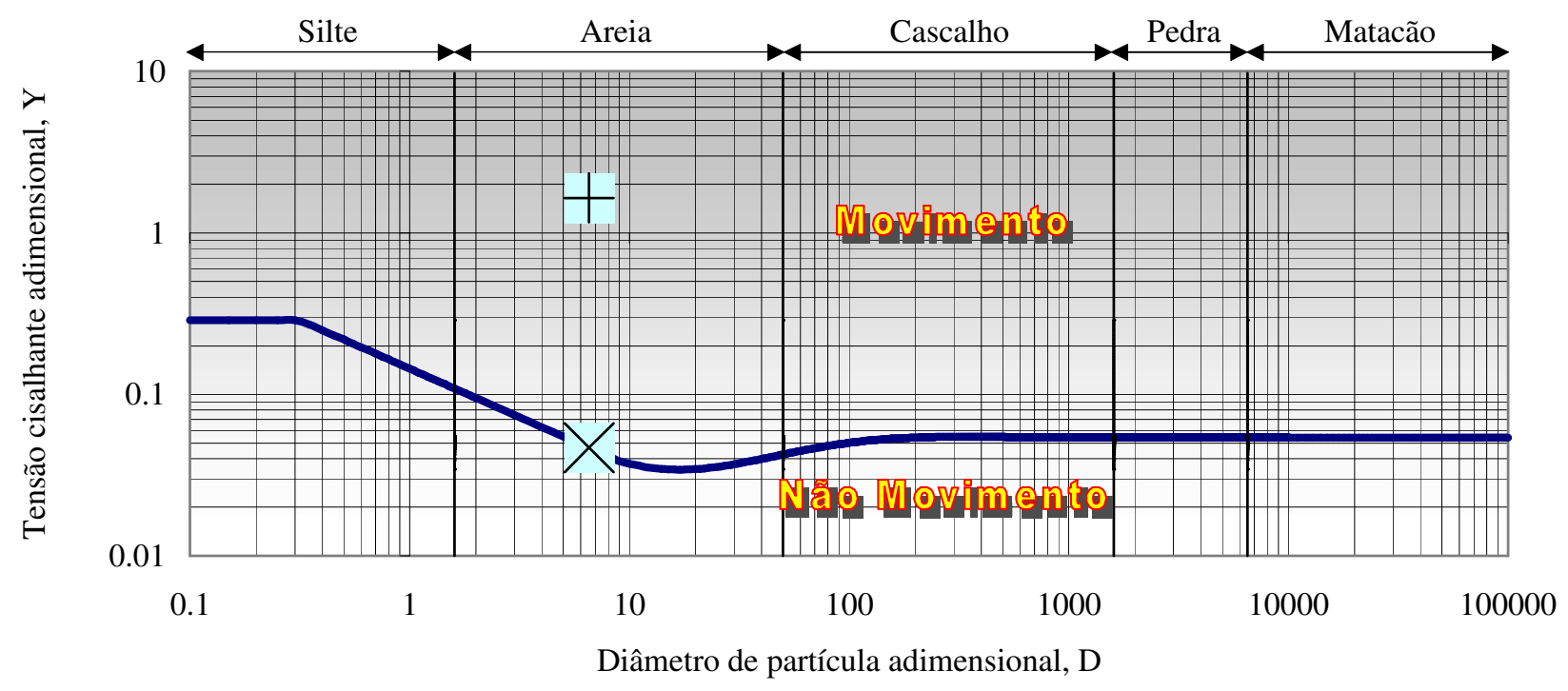

$+(\mathrm{D}=6,549 ; \mathrm{Y}=1,649) ; \quad X \quad(\mathrm{D}=6,549 ; \mathrm{Y}=0,047) ; \mathrm{e} \longrightarrow \quad$ Curva de Shields

FIGURA 2. Diagrama de Shields gerado com a rotina MSEY.

Na Figura 2, uma partícula com diâmetro de 0,000241 m ( $D=6,549)$, submetida a uma tensão cisalhante de 6,44 $\mathrm{Pa}(\mathrm{Y}=1,649)$, como determinado por MARTINS FILHO (1994), apresenta condições de iniciar movimento. Sobre a curva de Shields está grafado o limite de Y mínimo $\left(\mathrm{Y}_{\mathrm{cr}}=0,047\right)$ necessário para que tal partícula $(\mathrm{d}=0,000241 \mathrm{~m})$ inicie movimento. Portanto, sob essas condições e com o uso da rotina MSEY, foi possível determinar $\mathrm{T}_{\mathrm{c}}$ utilizandose da equação de Yalin para as 24 observações de MARTINS FILHO (1994). Desse modo, as seguintes equações foram calibradas:

$$
\begin{array}{ll}
\mathrm{T}_{\mathrm{c}}=0,04341 \tau^{1,614} & \mathrm{R}^{2}=0,95 \\
\mathrm{~T}_{\mathrm{c}}=0,05653 \tau^{3 / 2} & \mathrm{R}^{2}=0,95
\end{array}
$$

As eqs.(23) e (24) são aquelas ajustadas ou calibradas a partir dos resultados de $\mathrm{T}_{\mathrm{c}}$ obtidos com a rotina MSEY, utilizando-se dos dados obtidos por MARTINS FILHO (1994). A eq. (24) é uma calibração daquela proposta por FINKNER et al. (1989), na qual o expoente é fixado como sendo igual a 3/2. O expoente da eq.(23) é semelhante ao obtido por BRAIDA (1994), para um Argissolo no Rio Grande do Sul, que foi de 1,65. Já o coeficiente de transporte de sedimento determinado por BRAIDA (1994), foi de 0,05421, enquanto no presente trabalho foi de 0,04341 e $0,05653 \mathrm{com}$ as eqs.(23) e (24), respectivamente. Esses dois últimos coeficientes são da mesma ordem de grandeza daquele obtido por BRAIDA (1994).

A capacidade média de transporte de sedimentos pelo fluxo e a relação da carga e capacidade de transporte de sedimentos (G/T $\mathrm{T}_{\mathrm{c}}$ ), utilizando-se das eqs.(23), (24) e (7) do MSEY, para valores obtidos por PEREIRA (1989), são apresentadas na Tabela 3. Valores de $T_{c}$ e $G / T_{c}$ estimados com as eqs. (23) e (24) não diferiram significativamente, pelo teste Tukey, a 5\% de probabilidade, 
daqueles obtidos com a eq.(7), que é a equação de Yalin solucionada com o MSEY. Tal resultado expressa, portanto, a possibilidade de uso das eqs.(23) e (24) nas estimativas de $\mathrm{T}_{\mathrm{c}}$ em substituição à eq.(7).

TABELA 3. Valores de capacidade de transporte de sedimentos obtidos para os resultados de PEREIRA (1989).

\begin{tabular}{ccccccc}
\hline$\lambda$ & $\begin{array}{c}\mathrm{T}_{\mathrm{c}} \\
\text { eq.(23) }\end{array}$ & $\begin{array}{c}\mathrm{T}_{\mathrm{c}} \\
\text { eq. }(24)\end{array}$ & $\begin{array}{c}\mathrm{T}_{\mathrm{c}} \\
\text { eq.( }(7)\end{array}$ & $\begin{array}{c}\mathrm{G} / \mathrm{T}_{\mathrm{c}} \\
\text { eq. }(23)\end{array}$ & $\begin{array}{c}\mathrm{G} / \mathrm{T}_{\mathrm{c}} \\
\text { eq.(24) }\end{array}$ & $\begin{array}{c}\mathrm{G} / \mathrm{T}_{\mathrm{c}} \\
\text { eq.(7) }\end{array}$ \\
\hline$(\mathrm{m})$ & ----------- & $\mathrm{kg} \mathrm{m} \mathrm{s}^{-1} \mathrm{~s}^{-1}$ & ---------- & & & \\
\hline 1,0 & $2,355 \mathrm{cA}$ & $2,309 \mathrm{cA}$ & $2,435 \mathrm{Ca}$ & $0,00001 \mathrm{cA}$ & $0,00001 \mathrm{cA}$ & $0,00001 \mathrm{cA}$ \\
2,0 & $2,368 \mathrm{cA}$ & $2,313 \mathrm{cA}$ & $2,474 \mathrm{cA}$ & $0,00005 \mathrm{cA}$ & $0,00005 \mathrm{cA}$ & $0,00005 \mathrm{cA}$ \\
4,0 & $5,917 \mathrm{bA}$ & $5,427 \mathrm{bA}$ & $6,969 \mathrm{bA}$ & $0,00027 \mathrm{bA}$ & $0,00027 \mathrm{bA}$ & $0,00022 \mathrm{bA}$ \\
8,0 & $10,175 \mathrm{aA}$ & $8,930 \mathrm{aA}$ & $11,783 \mathrm{aA}$ & $0,00059 \mathrm{aA}$ & $0,00061 \mathrm{aA}$ & $0,00051 \mathrm{aA}$ \\
\hline
\end{tabular}

$\lambda$ - comprimento do declive; $\mathrm{T}_{\mathrm{c}}$ - capacidade de transporte de sedimento; $\mathrm{G}$ - carga de sedimento; números entre parênteses indicam as equações utilizadas para estimar $\mathrm{T}_{\mathrm{c}}$; médias seguidas de mesma letra minúscula na coluna e maiúscula na linha não diferem, significativamente, pelo teste de Tukey, a 5\% de probabilidade.

Verifica-se que tanto a capacidade de transporte quanto a relação $\mathrm{G} / \mathrm{T}_{\mathrm{c}}$ variaram significativamente em função do comprimento do declive. Isso era esperado, uma vez que aumentando-se o comprimento do declive, aumenta-se tanto a capacidade de transporte quanto a carga média de sedimentos no fluxo. Esses resultados corroboram BRAIDA (1994).

A relação $\mathrm{G} / \mathrm{T}_{\mathrm{c}}$ indica quanto da capacidade de transporte do fluxo está sendo ocupada com o transporte de sedimentos. No presente trabalho, verificou-se que a carga média de sedimentos transportados pelo fluxo foi sempre inferior à da sua capacidade de transporte, independentemente do método utilizado para determinar $\mathrm{T}_{\mathrm{c}}$. Assim sendo, foi possível assumir que a taxa de desagregação do solo durante os testes realizados por PEREIRA (1989) foi muito semelhante à capacidade de desagregação do fluxo, ou seja, que o termo $1-\mathrm{G} / \mathrm{T}_{\mathrm{c}}$ da eq.(5) tendeu para a unidade. Estes resultados também condizem com aqueles obtidos por BRAIDA (1994).

$\mathrm{Na}$ Tabela 4, apresentam-se parâmetros estatísticos propostos por LOAGUE \& GREEN (1991), os quais são oriundos das comparações dos valores de $T_{c}$ obtidos com as eqs.(23) e (24) com aqueles obtidos com a eq.(7), aplicando as eqs.(20) a (22).

TABELA 4. Parâmetros estatísticos do desempenho das eqs.(23) e (24) para a predição da capacidade de transporte de sedimentos num Latossolo Vermelho.

\begin{tabular}{cccc}
\hline Equação & RMSE (\%) & EF & CRM \\
\hline$(23)$ & 17,87 & 0,95 & 0,13 \\
$(24)$ & 30,77 & 0,83 & 0,21 \\
\hline
\end{tabular}

RMSE - erro-padrão da estimativa normalizado; EF - eficiência do modelo; CRM - coeficiente de massa residual.

Quanto à eficiência das eqs.(23) e (24), pôde-se inferir que a eq.(23) foi mais eficiente na predição da capacidade de transporte de sedimentos. RISSE et al. (1993) afirmaram que o parâmetro EF compara valores observados à linha 1:1, na qual valores medidos e estimados são iguais. Valor igual a 1,0 para EF indica modelo perfeito (MARTINS FILHO, 1999), o que não é o caso dos valores apresentados na Tabela 4.

Segundo LOAGUE \& GREEN (1991), valores de CRM maiores do que zero indicam que os valores observados de $T_{c}$ são subestimados pelos valores preditos, pelo uso das eqs.(23) e (24). O CRM menor da eq.(23) permite inferir que ela é melhor do que a eq.(24) para estimativas de $\mathrm{T}_{\mathrm{c}}$ (Tabela 4).

Os erros-padrão da capacidade de transporte de sedimentos, quando do uso das eq.(23) e (24), foram baseados no RMSE, o qual representa o erro-padrão das estimativas normalizado 
(Tabela 4). O RMSE para a eq.(23) foi de 17,87\%, enquanto para a eq.(24) foi de 30,77\%. Observa-se, portanto, que a ordem de magnitude dos erros cometidos, ao se estimar os valores observados pelo uso da eq.(23), foi menor do que com a eq.(24). Em função do exposto, pôde-se inferir que existe maior confiabilidade e acuracidade das estimativas obtidas para $\mathrm{T}_{\mathrm{c}}$ quando do uso da eq.(23).

\section{CONCLUSÕES}

A rotina desenvolvida MSEY (Modelo de Solução da Equação de Yalin) demonstrou ser uma ferramenta viável para a solução rápida da equação de Yalin e do diagrama de Shields, quando da determinação da capacidade de transporte de sedimentos.

Testes de calibração e validação, com dados independentes, permitem afirmar que é possível utilizar uma equação simplificada, equivalente à equação de Yalin, para estimar a capacidade de transporte de sedimentos $\left(\mathrm{T}_{\mathrm{c}}\right)$ do Latossolo Vermelho distrófico estudado, a partir da tensão cisalhante do fluxo $(\tau)$, como: $T_{c}=0,04341 \tau^{1,6144}\left(R^{2}=0,95\right)$.

\section{REFERÊNCIAS}

ALONSO, C.V.; NEIBLING, W.H.; FOSTER, G.R. Estimating sediment transport capacity in watershed modeling. Transactions of the ASAE, St. Joseph, v.24, n.5, p.1211-20, 1981.

BRAIDA, J.A. Relações da erosão entressulcos, com resíduos vegetais em cobertura e erosão em sulcos em solo podzólico vermelho-escuro. 1994. 152 f. Dissertação (Mestrado em Ciência do Solo) - Universidade Federal do Rio Grande do Sul, Porto Alegre, 1994.

FINKNER, S.C.; NEARING, M.A.; FOSTER, G.R.; GILLEY, J.E. A simplified equation for modeling sediment transport capacity. Transactions of the ASAE, St. Joseph, v.32, n.5, p.1545-50, 1989.

FOSTER, G.R. Modeling the erosion process. In: HAAN, C.T.; JOHNSON, H.P.;

BRAKENSIEK, D.L. Hydrologic modeling of small watersheds. St. Joseph: American Society of Agricultural Engineers, 1982. p.296-380. (ASAE. Monograph, 5)

FOSTER, G.R.; LANE, L.J.; NEARING, M.A.; FINKNER, S.C.; FLANAGAN, D.C. In: LANE, L.J.; NEARING, M.A. (Eds.) USDA - Water erosion prediction project: hillslope profile model documentation. West Lafayette: National Soil Research Laboratory \& USDA - Agricultural Research Service, 1989. p.10.1-10.12. (NSREL. Report, 2).

GILLEY, J.E.; DORAN, J.W. Soil erosion potential former conservation reserve program sites. Transactions of the ASAE, St. Joseph, v.41, n.1, p.97-103, 1998.

GILLEY, J.E.; KOTTWITZ E.R.; SIMATION J.R. Hydraulic characteristics of rills. Transactions of the ASAE, St. Joseph, v.33, n.6, p.1900-6, 1990.

JULIEN, P.Y. Erosion and sedimentation. Cambridge: Cambridge University Press, 1998. 280 p.

LEI, T.W.; ZHANG, Q.; ZHAO, J.; TANG, Z. A laboratory study of sediment transport capacity in the dynamic process of rill erosion. Transactions of the ASAE, St. Joseph, v.44 n.6, p.1537-42, 2001.

LOAGUE, K.; GREEN, R.E. Statistical and graphical methods for evaluating solute transport models: overview and application. Journal of Contaminant Hydrology, Amsterdam, v.7, n.1, p.5173, Jan. 1991.

LU, J.K.; CASSOL, E.A.; MOLDENHAUER, W.C. Sediment transport relationships for sand and silt loam soils. Transactions of the ASAE, St. Joseph, v.32, n.6, p.1923-31, 2001.

MARTINS FILHO, M.V. Erodibilidade inter e intra-sulcos dum latossolo vermelho-escuro textura argilosa da região de Jaboticabal - SP. 1994. 143 f. Dissertação (Mestrado em Solos e Nutrição 
de Plantas) - Escola Superior de Agricultura "Luiz de Queiroz", Universidade de São Paulo, Piracicaba, 1994.

MARTINS FILHO, M.V. Modelagem do processo de erosão entressulcos em latossolos de Jaboticabal - SP, 1999. 140 f. Tese (Doutorado em Solos e Nutrição de Plantas) - Universidade Federal de Lavras, Lavras, 1999.

NEARING, M.A.; FOSTER, G.R.; LANE, L.J.; FINKNER, S.C. A process-based soil erosion model for USDA-Water erosion prediction project technology. Transactions of the ASAE, St. Joseph, v.32, n.5, p.1587-93, 1989.

PAPHITIS, D. Sediment movement under unidiretional flows: na assessment of empirical threshold curves. Coastal Engineering, Amsterdam, v.43, n.1, p.227-45, 2001.

PEREIRA, V.P. Perda de solo em Latossolo Vermelho-Escuro, textura argilosa, devida a impacto de gotas de chuva simulada e a comprimento de rampa. 1989. 106 f. Tese (Livre-Docência em Conservação do Solo) - Faculdade de Ciências Agrárias e Veterinárias, Universidade Estadual Paulista, Jaboticabal, 1989.

POLYAKOV, V.O.; NEARING, M.A. Sediment transport in rill flow under deposition and detachment conditions. Catena, Amsterdam, v.51, n.1 , p.33-43, 2003.

RISSE, L.M.; NEARING, M.A.; NICKS, A.D.; LAFLEN, J.M. Error assessment in the universal soil loss equation. Soil Science Society of America Proceedings, Madison, v.57, n.3, p.825-33, May/June 1993.

SHARMA, K.D.; MURTHY, J.S.R.; DHIR, R.P. Modeling sediment delivery in arid upland basins. Transactions of the ASAE, St. Joseph, v.39, n.2, p.517-24, 1996.

STATSOFT. Statistica: Quick reference. Tulsa: StatSoft, 1994. 148 p.

YALIN, Y.S. An expression for bed load transportation. Journal of Hydraulic Division, St. Joseph, v.3, n.1, p.221-50, 1963. 